\title{
AN EFFICIENT SAMPLING SCHEME FOR APPROXIMATE PROCESSING OF DECISION SUPPORT QUERIES
}

\author{
Amit Rudra ${ }^{1}$, Raj P. Gopalan ${ }^{2}$ and N. R. Achuthan ${ }^{3}$ \\ ${ }^{I}$ School of Information Systems, Curtin University, Kent Street, Bentley WA 6155, Australia \\ ${ }^{2}$ Department of Computing ,Curtin University, Kent Street, Bentley WA 6155, Australia \\ ${ }^{3}$ Department of Mathematics and Statistics, Curtin University, Kent Street, Bentley WA 6155, Australia \\ \{A.Rudra, R.Gopalan, N.R.Achuthan\}@curtin.edu.au
}

Keywords: Sampling, Approximate Query Processing, Data Warehousing.

\begin{abstract}
Decision support queries usually involve accessing enormous amount of data requiring significant retrieval time. Faster retrieval of query results can often save precious time for the decision maker. Pre-computation of materialised views and sampling are two ways of achieving significant speed up. However, drawing random samples for queries on range restricted attributes has two problems: small random samples may miss relevant records and drawing larger samples from disk can be inefficient due to the large number of disk accesses required. In this paper, we propose an efficient indexing scheme for quickly drawing relevant samples for data warehouse queries as well as propose the concepts of database and sample relevancy ratios. We describe a method for estimating query results for range restricted queries using this index and experimentally evaluate the scheme using a relatively large real dataset. Further, we compute the confidence intervals for the estimates to investigate whether the results can be guaranteed to be within the desired level of confidence. Our experiments on data from a retail data warehouse show promising results. We also report the levels of accuracy achieved for various types of aggregate queries and relate them to the database relevancy ratios of the queries.
\end{abstract}

\section{INTRODUCTION}

Analytical queries containing aggregate functions such as sum and average on a data warehouse are used to gain a good sense of the business situation and to support business decisions. Most often we require timely retrieval of query results with an acceptable level of accuracy rather than absolute precision, and so approximate results within certain limits of accuracy will be acceptable to the user. Precomputation with materialized views and sampling are two ways to handle such queries. However, it is impractical to maintain a large number of materialized views for all possible combinations of information retrieval (Hellerstein et al., 1997). In contrast, sampling can provide faster results that are accurate within given assured confidence levels.

The main motivation for use of sampling in processing queries on a large database or a data warehouse is to save time and resources. Even though random sampling is both efficient and effective as an approximation method, its use for database querying has attracted significant research interest only recently ( $\mathrm{Li}$ et al, 2008; Joshi and Jermaine, 2008; Jin et al., 2006). Sampling has also been shown to be effective for aggregate queries (Hellerstein et al., 1997; Jermaine, 2007; Jin et al., 2006; Jermaine, 2003; Jermaine et al., 2007). As sampling data may not be fully representative of the entire data in a data warehouse, it is desirable to return both the query result and the confidence intervals that indicate the reliability of the results $(\mathrm{Li}$ et al, 2008).

A significant problem with random sampling for database queries from a database on stored disk is that picking records at random requires almost the same amount of $\mathrm{I} / \mathrm{O}$ as processing the query over the whole database (Olken and Rotem, 1990). To keep down the cost of sampling based query processing, a more efficient method of drawing samples is needed. Another problem is that a random sample drawn from a very large dataset may not contain relevant records that satisfy the range restrictions of a given query. To deal with this problem, we require a 
sampling scheme that will include in the sample records satisfying the query predicates.

Joshi and Jermaine (2008) introduced the ACE Tree which is a binary tree index structure for efficiently drawing samples for processing database queries. They demonstrated the effectiveness of this structure for single and two attribute database queries, but did not deal with multi-attribute aggregate queries. For extending the ACE Tree to $k$ key attributes, Joshi and Jermaine proposed binary splitting of one attribute range after another at consecutive levels of the binary tree starting from the root; from level $k+1$, the process is repeated with each attribute in the same sequence as before. This process could lead to an index tree of very large height for a data warehouse even if only a relatively small number of attributes are considered.

Li et al. (2008) proposed a sampling cube framework for answering analytical queries on a data warehouse which calculates confidence intervals for any multidimensional query. The sampling cube is constructed from a random sample of the data warehouse. After building the sampling cube, there is no further access to the original data records should a query require a different sample from the one already drawn. If a query has too few sample records in the sampling cube, they expand the query to gather more sample records from the sampling cube itself in an attempt to improve the quality of the query result.

In this paper, we propose the $k$-MDI Tree which extends the ACE Tree structure to deal with multidimensional data warehouse queries. Unlike the ACE Tree, the $k$-MDI tree allows non-binary splits of data ranges for key values that do not split evenly into $2^{\mathrm{n}}$ distinct ranges. The number of levels in the $k$ MDI tree can be limited to the number of key attributes. The shallow tree structure resulting from multi-way branching also facilitates quicker retrieval of leaf nodes from disk storage. Unlike the sampling cube of $\mathrm{Li}$ et al. (2008), new samples that contain relevant records are drawn for each query. These records can be considered as drawn from a subset of the data warehouse that satisfies the query predicates. In estimating the query results, we take into account the proportion of relevant records for the query in the whole data warehouse. The sampling and estimation methods are evaluated experimentally using a real life data set.

The rest of the paper is organized as follows: In Section 2, we define some relevant terms and briefly describe the ACE Tree structure. In Section 3, our kway multi-dimensional $(k$-MDI) indexing structure is described in detail. We also introduce the concept of relevancy ratios, both for the database and a specific sample. Section 4 reports the experimental results that evaluate the efficacy of our scheme. Section 5 is the conclusion of the paper.

\section{TERMS, DEFINITIONS AND ACE TREE STRUCTURE}

In this section, we define some terms pertaining to data warehousing, define confidence interval and then review briefly the ACE Tree structure (Joshi and Jermaine, 2008) that has preceded the $k$-MDI tree we propose in Section 3.

\subsection{Dimensions and Measure}

To support decision support queries, data is usually structured in large databases called data warehouses. Typically, data warehouses are relational databases with a large table in the middle called the fact table connected to other tables called dimensions. For example, consider the fact table Sales shown as Table 1. A dimension table Store linked to StoreNo in this fact table will contain more information on each of the stores such as store name, location, state, and country (Hobbs, Hillson and Lawande, 2003). Other dimension tables could exist for items and date. The remaining attributes like quantity and amount are typically, but not necessarily, numerical and are termed measures. A typical decision support query aggregates a measure using functions such as Sum(), Avg() or Count(). The fact table Sales along with all its dimension tables form a star schema.

Table 1: Fact table Sales

\begin{tabular}{|c|l|l|r|r|}
\hline \multicolumn{5}{|c|}{ SALES } \\
\hline $\begin{array}{l}\text { Store } \\
\text { No }\end{array}$ & Date & Item & Quantity & Amount \\
\hline 21 & 12-Jan-11 & iPad & 223 & 123,455 \\
\hline 21 & 12-Jan-11 & PC & 20 & 24,800 \\
\hline 24 & 11-Jan-11 & iMac & 11 & 9,990 \\
\hline 77 & 25-Jan-11 & PC & 10 & 12,600 \\
\hline
\end{tabular}

In decision support queries a measure is of interest for calculation of averages, totals and counts. For example, a sales manager may like to know the total sales quantity and amount for certain item(s) in a certain period of time for a particular store or even all (or some) stores in a region. This may then allow her to make decisions to order more or less stocks as appropriate at a point in time. 


\subsection{Confidence Interval}

When estimating with samples we indicate the reliability of the estimate by its confidence interval. Consider a sample of records $x$ with the mean of the sample denoted by $\bar{x}$, and the size of the sample $n$. For a desired confidence level (e.g. 95\%) the confidence interval estimator of the population mean $\mu$ is given by:

$$
\bar{x}-t_{\alpha / 2} \frac{s}{\sqrt{\mathrm{n}}}, \quad \bar{x}+t_{\alpha / 2} \frac{s}{\sqrt{\mathrm{n}}}
$$

where $t_{\alpha / 2}$ is the critical t-value and $s$ the standard deviation of the sample (Keller, 2009).

\subsection{ACE Tree Structure}

The ACE Tree is a balanced binary tree where the leaf nodes contain the randomized samples of key values and the internal nodes above them are the index nodes. Each internal node contains a range $\mathrm{R}$ of key values, a key value $k$ that splits $\mathrm{R}$ into left and right sub-trees, pointers to the left and right branch (child) nodes, and counts of database records falling in the left and right sub-trees. Figure 1 shows the structure of an example ACE Tree. The root node $I_{1,1}$ with its range $I_{1,1} \cdot R$ labeled as [0-64] signifies the key value range of the whole data set. The key of the root node partitions the range $I_{1,1} \cdot R$ into $I_{2,1} \cdot R=[0$ 32] and $I_{2,2} \cdot R=$ [33-64]. This partitioning of ranges is propagated down the tree among the descendants of respective nodes. The ranges associated with a section of a leaf node are determined by the ranges associated with each internal node on the path from the root node to the leaf. If we look at the path from $I_{1,1}$ i.e. the root node down to the leaf node $L_{4}$, we come across the following ranges $0-64,0-32$ and 1724. A leaf node is partitioned into sections $\left(S_{1}, S_{2}\right.$, $\ldots)$, their number depending on the number of dimensions indexed. Thus, the first section $L_{4} \cdot S_{1}$ has a random sample of records in the range $0-64 ; L_{4} \cdot S_{2}$ has them in the range $0-32 ; L_{4} . S_{3}$ in the range $17-32$ and $L_{4} \cdot S_{4}$ in the range $25-32$. The size of each leaf is chosen as the number of records that can be stored in a disk block and so the number of leaf nodes depends on the size of the database which also determines the height of the index tree itself.

\subsection{Sampling for a Query Using the ACE Tree}

Referring to Figure 1, consider a query $Q$ with a range of [28-38]. The query execution algorithm proceeds by traversing down $I_{1,1}$, the root node. Both $I_{2,1} \cdot R$ and $I_{2,2} \cdot R$ overlaps with $Q$.

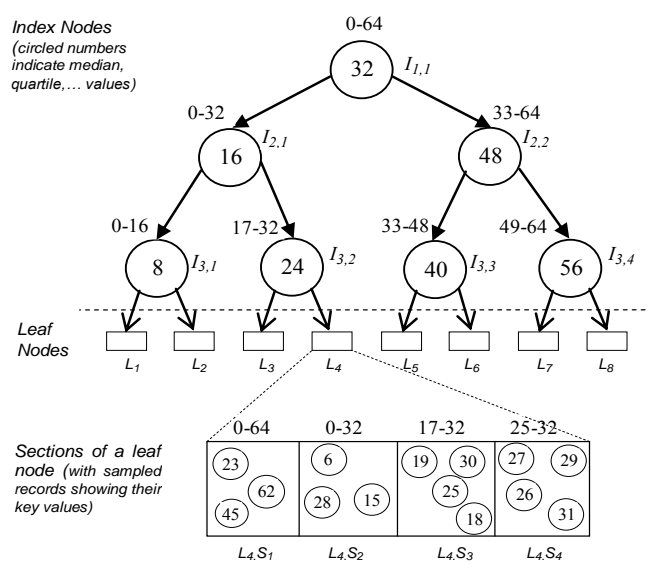

Figure 1: Structure of the ACE Tree

A level down from $I_{2,1}$, only $I_{3,2} \cdot R$, overlaps with $Q$. Traversing down to the leaf nodes, the algorithm finds the right leaf node's range [25-32] overlaps with $Q$ and so retrieves records from $L_{4}$. The relevant records in the query's range are returned for the sample which includes record 28 from $L_{4} . S_{2}$, record 30 from $L_{4} \cdot S_{3}$ and records 29 and 31 from $L_{4} . S_{4}$. Next, the algorithm traverses down the right node $I_{2,2}$ below the root to the leaf node $L_{5}$ and retrieves all relevant records from all sections of $L_{5}$ to the pool of sample records.

\subsection{Extended ACE Tree for Multiple Dimensions}

Joshi and Jermaine (2008) proposed extending the ACE Tree from a single dimension to multiple dimensions as follows: Given key attributes (dimensions), $a_{1}, \ldots, a_{\mathrm{k}}$, split the range of values for $a_{1}$ into two sub trees of approximately equal number of keys below the root (level 1); for each node at level 2 , similarly perform a binary split of the range of key values for $a_{2}$ and so on up to level $\mathrm{k}$ for attribute $a_{\mathrm{k}}$. Then at level $\mathrm{k}+1$, split the attribute values of $a_{1}$ again followed by $a_{2}$, etc. at further lower levels.

In real life data, a dimension's values may not split evenly into $2^{\mathrm{n}}$ distinct ranges. For example, if a dimension has an odd number of key values, say $k_{1}, k_{2}$ and $k_{3}$, with cardinalities of 30000 each; then, we cannot spit them evenly into 2 distinct ranges but we can do so into 3 . The height of the tree will be very large even for a moderate sized data warehouse with a relatively small number of dimensions. 


\section{MULTIDIMENSIONAL INDEXING}

We propose the $k$-MDI tree which extends the ACE Tree index for multiple dimensions while overcoming the limitations of the ACE Tree discussed in Section 2.5. The height of the $k$-MDI tree is limited to the number of key attributes. As a multi-way tree index, it is relatively shallow even for a large number of key value ranges and so requires only a small number of disk accesses to traverse from the root to the leaf nodes.

\section{1 $k$-ary Multidimensional Index $(k$ - MDI)}

The $k$-ary multi-dimensional index tree ( $k$-MDI tree) is a $k$-ary balanced tree as described below:

1. The root node of a $k$-MDI tree corresponds to the first attribute (dimension) in the index.

2. The root points to $k_{1}\left(k_{1} \leq k\right)$ index nodes at level 2 , with each node corresponding to one of the $k_{1}$ splits of the ranges for attribute $a_{1}$.

3. Each of the nodes at level 2, in turn, points to up to $k_{2} \quad\left(k_{2} \leq k\right)$ index nodes at level 3 corresponding to $k_{2}$ splits of the ranges of values of attribute $a_{2}$; similarly for nodes at levels 3 to $h$, corresponding to attributes $a_{3}, \ldots, a_{\mathrm{h}}$.

4. At level $h$, each of up to $k^{h-1}$ nodes points to up to $k_{\mathrm{h}}\left(k_{\mathrm{h}} \leq k\right)$ leaf nodes that store data records.

5. Each leaf node has $h+1$ sections; for sections 1 to $h$, each section $i$ contains random subset of records in the key range of the node $i$ in the path from the root to the level $h$ above the leaf; section $h+1$ contains a random subset of records with keys in the specific range for the given leaf. Thus, the dataset is divided into a maximum of $k^{h}$ leaf nodes with each leaf node, in turn, consisting of $h+1$ sections and each section containing a random subset of records. The total number of leaf nodes depends on the total number of records in the dataset and the size of a leaf node (which may be chosen as equal to the disk block size or another suitable size). More details on leaf nodes and sections are given in Section 3.3. In real data sets, the number of range splits at different nodes of a given level $i$ need not be the same. For convenience, the number of splits at all levels are kept as $k$ in Figure 2 that shows the structure of the general scheme for $k$-MDI multilevel index tree of attributes $\mathrm{A}_{1}, \mathrm{~A}_{2}, \ldots, \mathrm{A}_{\mathrm{h}}$ with $k$ ranges $\left(R_{11}, R_{12}, \ldots, R_{1 \mathrm{k}}\right),\left(R_{21}, R_{22}, \ldots, R_{2 \mathrm{k}}\right), \ldots\left(R_{\mathrm{h} 1}, R_{\mathrm{h} 2}, \ldots\right.$, $\left.R_{\mathrm{hk}}\right)$ respectively at levels $(1, \ldots, h)$.
An example of the $k$-MDI tree is shown in Figure 3 from a store chain dataset with three dimensions store, date sold and item number. The number of range splits and hence branches from non-leaf nodes vary between 2 and 4 in this example.

\subsection{Leaf Nodes}

Similar to the ACE tree structure, the lowest level nodes of a $k$-MDI tree point to leaf nodes containing data records. The data records are stored in $h+1$ sections, where $h$ is the height of the tree. Section $S_{1}$ of every leaf node is drawn from the entire database with no range restriction on the attribute values. Each section $S_{\mathrm{i}}(2 \leq i \leq h+1)$ in a leaf node $L$ is restricted on the range of key values by the same restrictions that apply to the corresponding sub-path along the path from the root to $L$. Thus for section $S_{2}$, the restrictions are the same as on the branch to the node at level 2 along the path from the root to $L$ and so on.

Figure 3 shows an example leaf node projected from the sample $k$-MDI tree. The sections are indicated above the node with attribute ranges for each section below the node. The circled numbers in each section indicate record numbers that are randomly placed in the section. The range restrictions on the records are indicated below each section, where the first section $S_{1}$ has records drawn from the entire range of the database. Thus, it can contain records uniformly sampled from the whole dataset. The next section $S_{2}$ has restriction on the first dimension viz. store (for leaf node $L_{7}$ this range is store numbers 1-16). The third section $S_{3}$ has restrictions on both first and second dimensions viz. store and date. While the last section $S_{4}$ has restrictions on all the three dimensions - store, date and item.

The scheme for selection of records into various leaf nodes and sections is explained in detail in the following section.

\subsection{Building the $k$-MDI Tree}

The purpose of the $k$-MDI tree is to quickly retrieve relevant random samples of records for processing data warehouse queries. The records in the sample are obtained from leaf nodes by traversing the index from the root. The $k$-MDI Tree is built in the following three steps:

1. First, the dataset records are sorted by the first key attribute $a_{1}$ as the major field, followed by the second attribute $a_{2}$ and so on until the last attribute $a_{\mathrm{h}}$. 
2. The next step is to find the split points of key attribute values in the index tree at the levels 1 to $h$ so that the number of records of the dataset that fall under each sub-tree rooted at levels 2 to $\mathrm{h}$ is approximately equal. The $k_{1}-1$ split points at level 1 are chosen such that the total number of records in the dataset are split into $k_{1}$ approximately equal parts; the records falling under each of the nodes at level 2 are split into $k_{2}$ approximately equal parts, and so on until the records falling under each of the nodes at level $h$ split into $k_{h}$ approximately equal parts. The number of splits at all the levels in the index should be such that the number of leaf nodes are equal to a pre-computed number based on the total number of records in the dataset and the size of each leaf node (which could be chosen as the disk block size as in the case of the ACE Tree or some other suitable size).

3. Next, a random number between 1 and $h+1$ is assigned to each data record as its section number. Depending on the section number and its composite key value, the record is assigned to a leaf node as follows: If the section number is 1 , the record is assigned randomly to any one of the leaf nodes in the tree; if the section number is $i(2 \leq i \leq h)$, starting from the root of the index tree, we locate the root of a sub-tree at level $i$ in which the key of the record falls and assign the record randomly to section $i$ of any of the leaf nodes in that sub-tree; if the record's section number is $h+1$, it is assigned to the specific leaf node where the record's key value belongs. When all the records have been thus assigned section and leaf node numbers, the dataset is re-organised with records sorted according to their leaf node and section numbers.

\subsection{Using the $k$-MDI Tree for Data Warehouse Queries}

By using a k-MDI tree index, we can draw stratified samples for data warehousing queries from restricted ranges of key values. In this section, we first introduce two measures that are useful for the estimation of query results using such samples. The database relevancy ratio (DRR) of a query $\mathrm{Q}$, denoted by $\rho(Q)$ is the ratio of the number of records in a dataset $\mathrm{D}$ that satisfies the query conditions to the total number of records in D. For a query with no condition, $\rho(Q)$ is 1 . Similarly, the sample relevancy ratio (SRR) of a query $\mathrm{Q}$ for a sample set $\mathrm{S}$, denoted by $\rho(\mathrm{Q}, \mathrm{S})$ is defined as the ratio of the number of records in $\mathrm{S}$ that satisfy a given query $\mathrm{Q}$ to the total number of records in $\mathrm{S}$.

In a true random sample of records, the SRR for a query $\mathrm{Q}$ is expected to be equal to its $\mathrm{DRR}$, i.e., $\mathrm{E}(\rho(\mathrm{Q}, \mathrm{S}))=\rho(\mathrm{Q})$. A sample with $\rho(\mathrm{Q}, \mathrm{S})>\rho(\mathrm{Q})$ is likely to give a better estimate of the mean than a true random sample. However, for the sum of a column, the sample needs to be representative of the population, i.e., $\rho(Q, S)$ should be close to $\rho(Q)$.

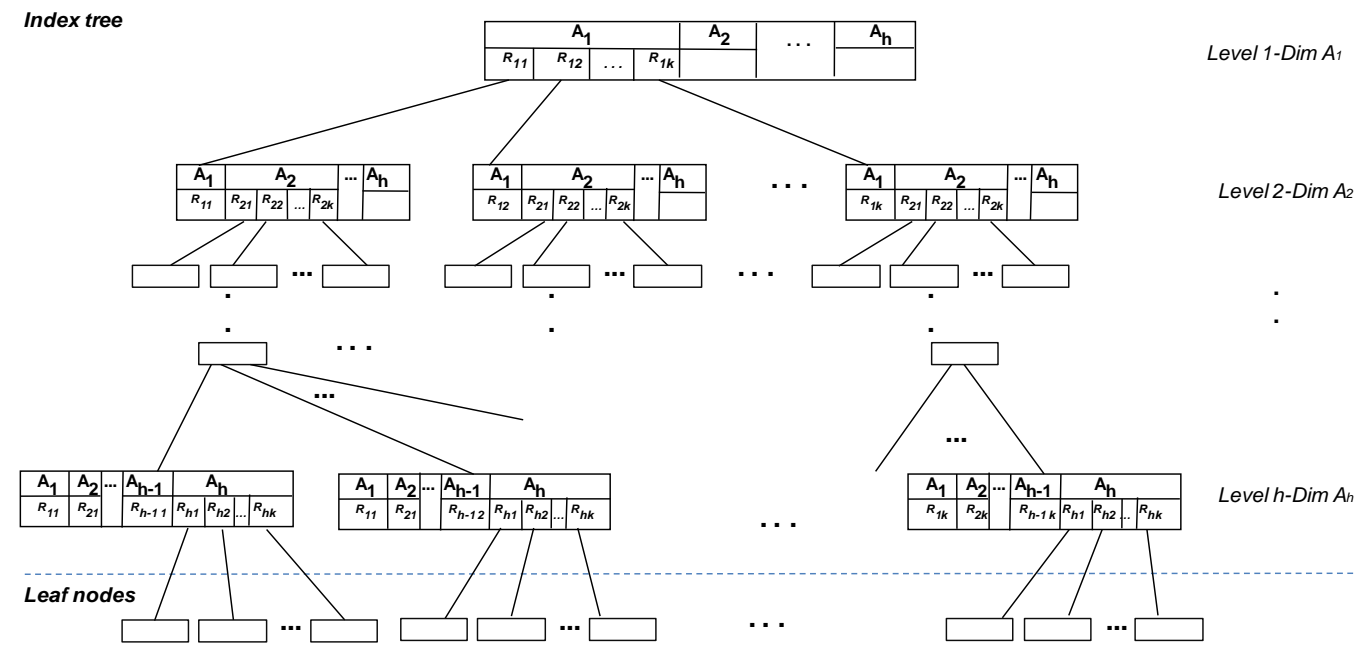

Figure 2: General structure of the k-MDI tree $-\mathrm{A}_{1}, \mathrm{~A}_{2}, \ldots, \mathrm{A}_{\mathrm{h}}$ are $\mathrm{h}$ attributes and $R_{i j}$ the $i$-th attribute's $j$-th range high water mark (HWM). 
Consider the following formula for estimating the sum (Berenson and Levine, 1992): $\widehat{T}=N \hat{p} \bar{X}$, where $N$ is the cardinality of the population, $\hat{p}$ the estimated proportion of records satisfying the query conditions and $\bar{X}$ the mean of records in the sample satisfying the query condition. In order to estimate the mean we can use all relevant sampled records from all sections of the retrieved leaf nodes, but to estimate the sum we can use sampled records only from section $S_{1}$, which is the only section with records drawn randomly from the entire dataset. For estimating the sum for a query with conditions on some of the indexed dimensions we use appropriate sections of the retrieved leaf nodes to get a better estimate of the mean; the records from section $S_{1}$ are also used to get a fair estimation of the proportion records that satisfy the query conditions.

\subsection{Effect of Sectioning on Relevancy Ratio}

As discussed earlier, sections $S_{1}$ to $S_{h+1}$ of each leaf node contain random collections of records with the difference that $S_{1}$ contains records from the entire dataset while other sections contain random records from restricted ranges of the key attributes. Consider a query with the same range restrictions on all three dimensions (store, date and item) as section $L_{7} . S_{4}$ in Figure 3 . We are then likely to get more relevant records in the sample from the second section $L_{7} . S_{2}$ than from $S_{1}$ since records of $S_{2}$ have restrictions on the first dimension of store that matches the query condition. Records in $S_{3}$ will have restrictions on both store and date dimensions that match that of the query and so are likely to contain more relevant records than in $S_{2}$. All records in section $L_{7} . S_{4}$ will satisfy the query since the range restrictions on $S_{4}$ exactly match the query. Mathematically, for a query $\mathrm{Q}$ having restrictions as mentioned above:

$$
\begin{aligned}
\rho(\mathrm{Q}) & =\mathrm{E}\left(\rho\left(\mathrm{Q}, L_{7} . S_{1}\right)\right) \leq \mathrm{E}\left(\rho\left(\mathrm{Q}, L_{7} \cdot S_{2}\right)\right) \\
& \leq \mathrm{E}\left(\rho\left(\mathrm{Q}, L_{7} . S_{3}\right)\right) \leq \mathrm{E}\left(\rho\left(\mathrm{Q}, L_{7} . S_{4}\right)\right)
\end{aligned}
$$

Using this property of the $k$-MDI tree, it is possible to quickly increase the size of a sample that is too small, by including more records from other sections of the retrieved leaf nodes.

\subsection{Record Retrieval to Process a Query}

The objective of using the $k$-MDI tree is to retrieve a significant number of relevant records (i.e. records that satisfy the query conditions) in the sample drawn for processing a given query. The query conditions may span sections of one or more leaf nodes which can be reached from index nodes that straddle more than one range of attribute values. These leaf nodes can be accessed by traversing the tree from the root using the attribute value ranges in the query conditions and sections from multiple leaf nodes can be combined to form the sample.

We describe the retrieval process using an example query on the sample database of Figure 4 Consider a query $\mathrm{Q}_{0}$ about sales in store 12 for date range $1-13$ and item range $12 \mathrm{M}-20 \mathrm{M}$. The retrieval algorithm finds the sections of leaf nodes for this query as follows:

1. Search index level 1 to locate the relevant store range. Store 12 is in the left most range of 1 16.

2. Traverse down to index level 2 (date), indicated by a dashed arrow in Figure 4, along the first store range. Since there is a condition on date (1-13), compare the HWMs (high water marks) of the three ranges and find that it fits into two date ranges viz. the first and the second. Make a note of these date ranges.

3. Traverse down using the first date range to the next index level which has item ranges. Since there is a condition on item numbers (12M20M), compare this range with HWMs and find that it fits into two ranges viz. the third and the fourth. Make a note of these item ranges.

4. Traverse down using the third item range to relevant leaf pages and make a note of them.

5. Iterate step 4, except this time using the fourth item range.

6. Next, repeat the above three steps i.e. steps 3 through 5; but this time using the second date range instead.

7. Now retrieve records from the relevant sections in the four leaf nodes (viz. $L_{3}, L_{4}, L_{7}$ and $L_{8}$ ) to form a sample for the given query. 


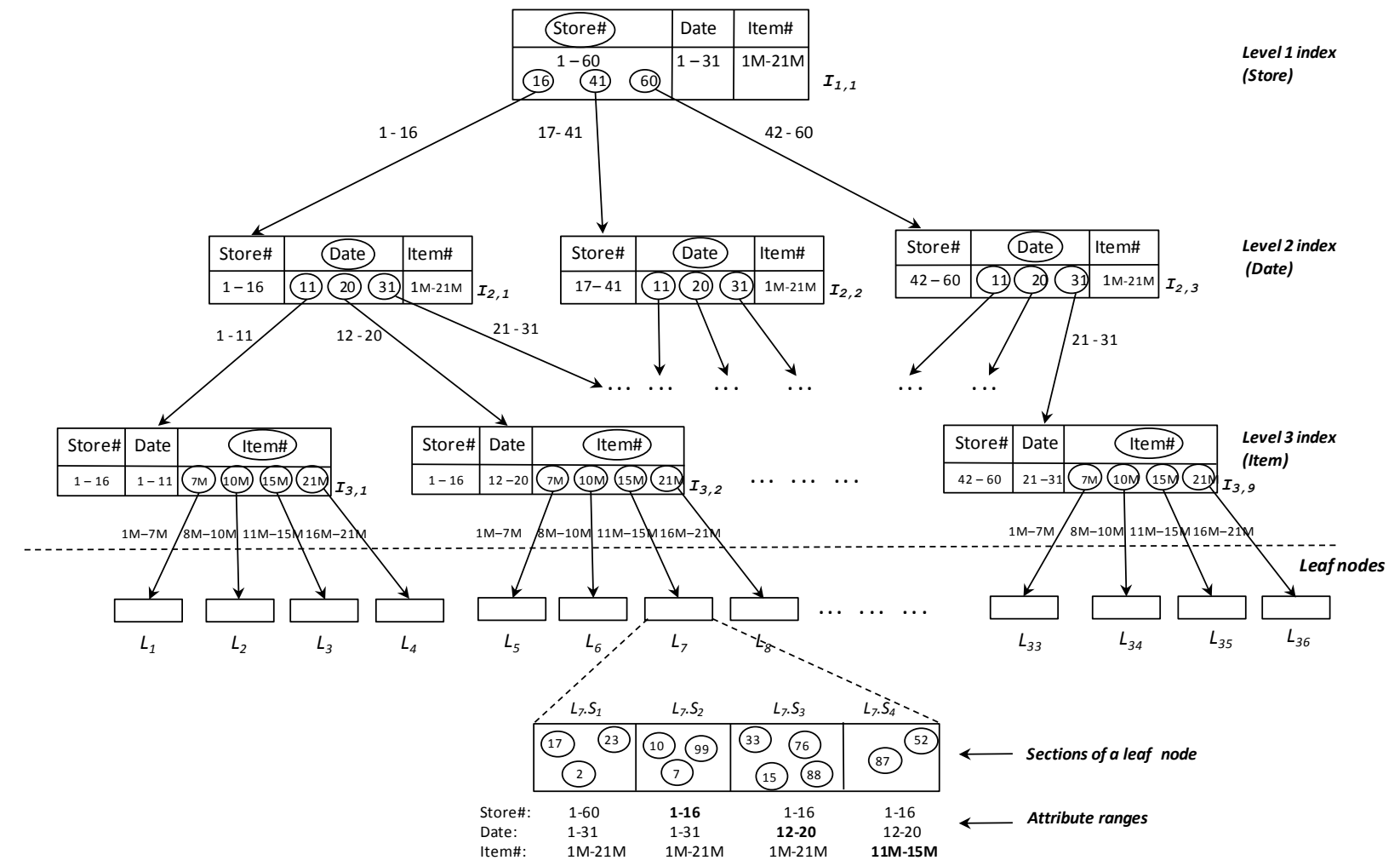

Figure 3: A leaf node (changes in range values for attributes are indicated in bold).

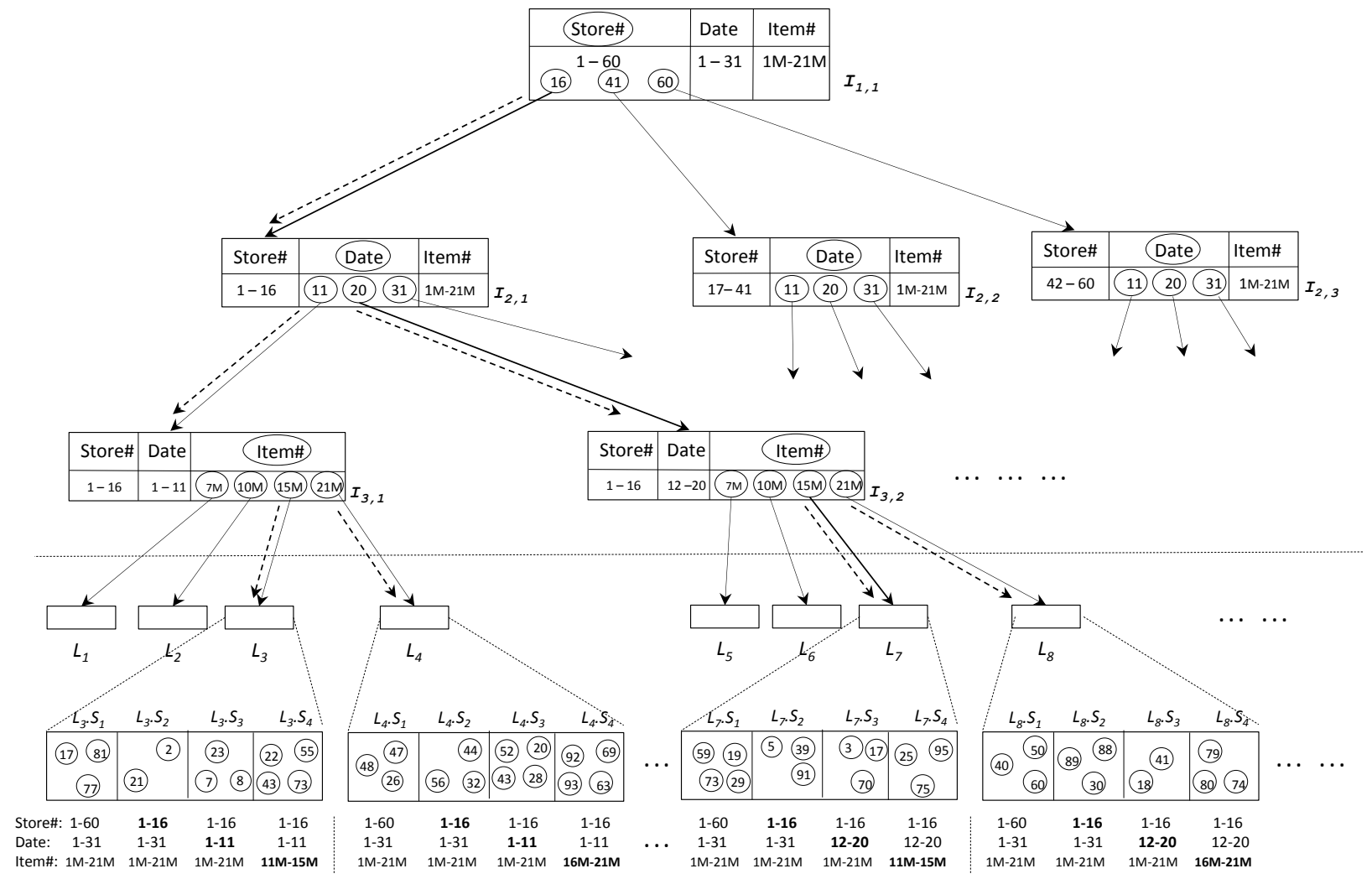

Figure 4: Navigation down index tree nodes for conditions on three dimensions 


\subsection{Estimating Query Results from Samples}

In decision support queries on large databases, the most common estimation performed is either of the mean or the sum of a column measure (Jin et al, 2006). We maintain a table representing a histogram of record counts for each leaf node and its sections. It is used to estimate the number the leaf nodes required to have adequate number of samples. The following steps outline our method of estimating the mean, sum, standard deviation and the confidence intervals:

1. Draw a sample set L of leaf nodes as described in Section 3.6 for the given sampling rate.

2. The following parameters are computed:

a. Sample size $-n$

b. Count of sampled records satisfying the query condition $-m$

c. Count of records in all sampled $\mathrm{S}_{1}$ sections of $\mathrm{L}-n^{\prime}$

d. Count of records in all sampled ${ }_{\mathrm{S} 1}$ sections of $\mathrm{L}$ satisfying the query condition $-m^{\prime}$

e. Sum of attribute (variable) value of all $\mathrm{m}$ records - sum

f. Sum of squares of attribute value of the all abovementioned $m^{\prime}$ records $-s u m S q^{\prime}$

g. The average sum of squares $-Z=\frac{s u m s q^{\prime}}{\dot{m}}$

3. Estimating the sum, average, variance and C.I. limits (Chaudhuri and Mukherjee, 1985):

a. Estimate of the number of records $M$ that satisfy the query condition in the population (given the cardinality of the dataset $N$ )

$$
\widehat{M}=N \frac{m^{\prime}-1}{n^{\prime}-1}
$$

b. Estimate of Average $\bar{x}=\frac{s u m}{m}$

c. Estimate of Sum $\widehat{T}=\widehat{M} \bar{x}$

d. Estimate of variance of Average $\mathrm{v}(\bar{x})$

$$
=\frac{\hat{M}-m}{\hat{M}(m-1)}\left(Z-\left(\bar{x}^{2}\right)\right)
$$

e. Confidence interval lower limit

$$
=\frac{m^{\prime}-1}{n^{\prime}-1}-Z \sqrt{\frac{\left(m^{\prime}-1\right)}{n\left(n^{\prime}-1\right)} \frac{\left(n^{\prime}-m^{\prime}\right)}{\left(n^{\prime}-1\right)}}
$$

f. Confidence interval upper limit

$$
=\frac{m^{\prime}-1}{n^{\prime}-1}+Z \sqrt{\frac{\left(m^{\prime}-1\right)}{n\left(n^{\prime}-1\right)} \frac{\left(n^{\prime}-m^{\prime}\right)}{\left(n^{\prime}-1\right)}}
$$

\section{EXPERIMENTAL RESULTS}

To evaluate the effectiveness of our sampling technique based on the $k$-MDI tree, we performed experiments on real life supermarket retail sales data (TUN, 2011) for a month from 150 outlets. The data warehouse is structured as a star schema shown in Figure 5, with the fact table (itemscan) consisting of over 21 million rows and three dimension tables viz. storeInfo, itemDesc and storeMemberVisits. TPC-H queries (TPC Benchmarks, 2011) with suitable modifications for this sample data warehouse were used in the experiments. Most of the TPC-H queries involve SQL aggregate functions of $\operatorname{sum}(), \operatorname{avg}()$ or count(). A few include $\min ()$ and $\max ()$ which are not easily calculated by sampling (Joshi, 2008; Hellerstein et al, 1997). So, we investigated only sum, avg and count in our experiments. The index tree structure was simulated using the Oracle DBMS. We clustered the fact table on leaf and section number to maintain the records in that sequence. This organisation supported the simulation of both the storage and retrieval of records for the experiments.

A set of three queries were used containing the SQL functions $-\operatorname{avg}(), \operatorname{sum}()$, count() with varying database relevancy ratios (DRR). The queries were of the form:

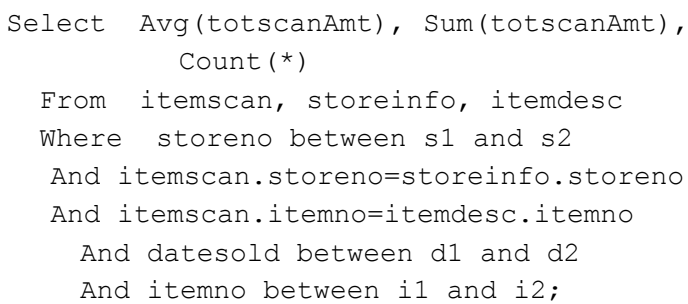

The DDR value was set high or low for the queries by choosing a given proportion of the dimension range for the query. For example, assuming a uniform distribution of values for a dimension in the database, we can get a DRR of approximately 0.33 on a single dimension query, by picking a third of the dimension range. However, in practice we empirically varied the dimension ranges in the queries to get the desired DRR values.

The first test query had a condition on a single dimension and a high DRR value of 0.37; the second query had a lower DRR (0.05) with conditions on two dimensions; and the third query had a very low DRR (0.002) with conditions on all three dimensions. The relevance of DRR in estimating the query results may be seen from the query result estimation process of Section 3.7. In step 3a, the 


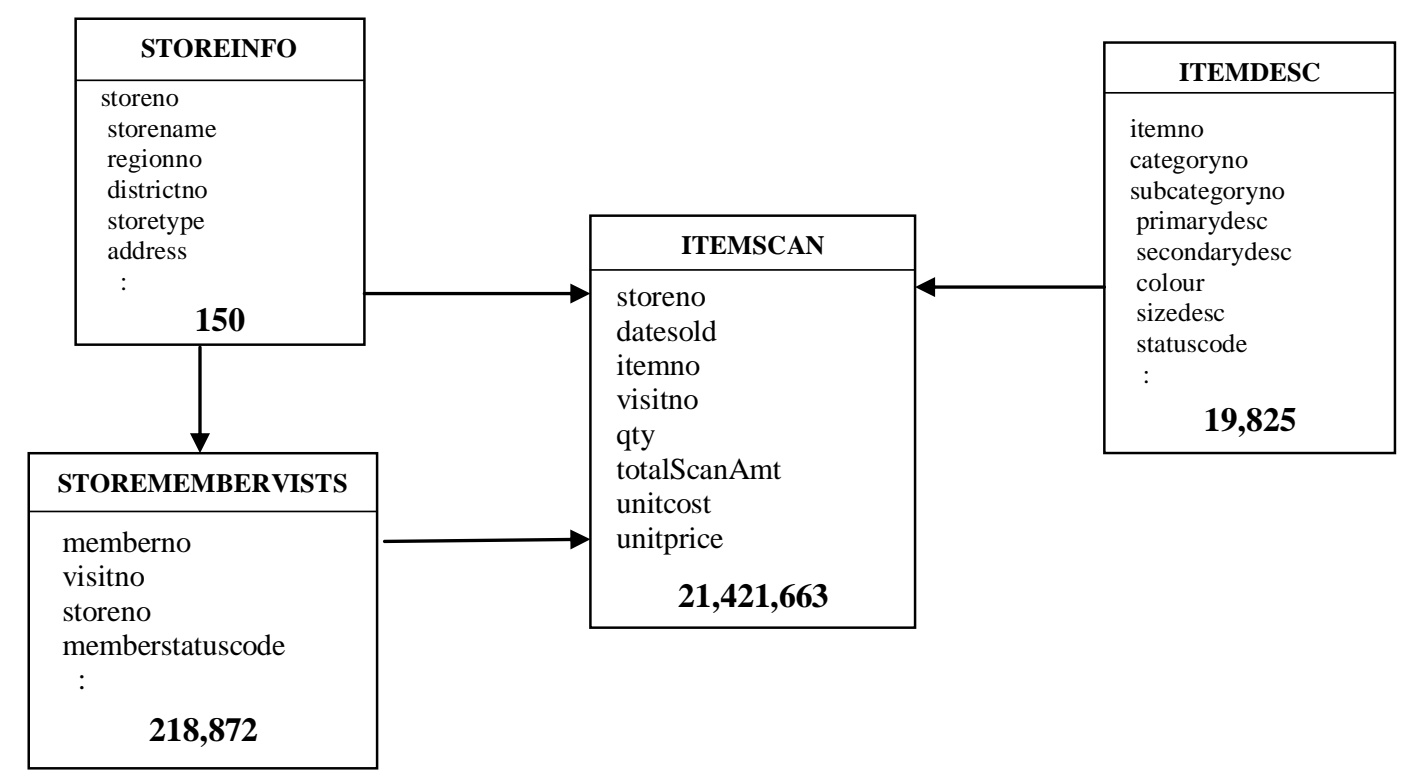

Figure 5: The schema for experimental retail sales data warehouse

count $\widehat{M}$ directly depends upon the DRR, which is the statistical proportion $p$ whose estimate is given by $\frac{\boldsymbol{m}^{\prime}-1}{\boldsymbol{n}^{\prime}-1}$. In step $3 \mathrm{c}$, the estimation of sum depends on the count $\widehat{M}$ and thereby on $p$.

We conducted the experiments using several random samples at sampling rates of $(1 \%-12 \%)$ and the results were averaged for each sampling rate. The error for the three aggregate functions viz. avg, sum and count were computed as the absolute value of the difference between estimated and actual values for the whole database. Figure 6 shows the results with error rates for both average (mean) of totscanamt column, sum of totscanamt and count for the different database relevancy ratios mentioned above.

There are two graphs for each level of DRR. Figure 6a shows the error rates for the average, the sum of scan amount and count for high DRR. Figure $6 \mathrm{~b}$ shows the confidence intervals (lower and upper limits) for the average amount for high DRR. Figure $6 \mathrm{~b}$ also shows the estimated and actual values of the average scan amount. Figures $6 c$ and $6 d$ show similar information as above for the low value of DRR; Figures 6e and $6 f$ show similar information for very low DRR.
It is seen that for the high DRR query with $\rho\left(Q_{1}\right)$ $=0.37$, the error rates for the count, average and the sum of the total scan amount stabilize as the sampling rate is increased. The estimates are close to the actual values for the lowest to the highest sampling rates used and the true value of average is always within the estimated confidence interval. For the medium DRR query with $\rho\left(Q_{1}\right)=0.05$, we still get error rates below the normally acceptable rate of $5 \%$. For query with very low DRR of $\rho\left(Q_{1}\right)=0.002$, the error rates for the average scan amount, for all but $1 \%$ sampling rate, are below $5 \%$ and the true values within the C.I. limits. But the error rates for the estimated sum of scan amount and count of records are not below the acceptable limit at any sampling rate used for the very low DRR query. Thus, we cannot satisfactorily estimate the sum and the count for low values of DRR, while for medium to high values of DRR the estimations of both the sum and average are within acceptable error limits. Also, it's observed from the graphs that there is an apparent close correlation between the estimates of sum and count. 


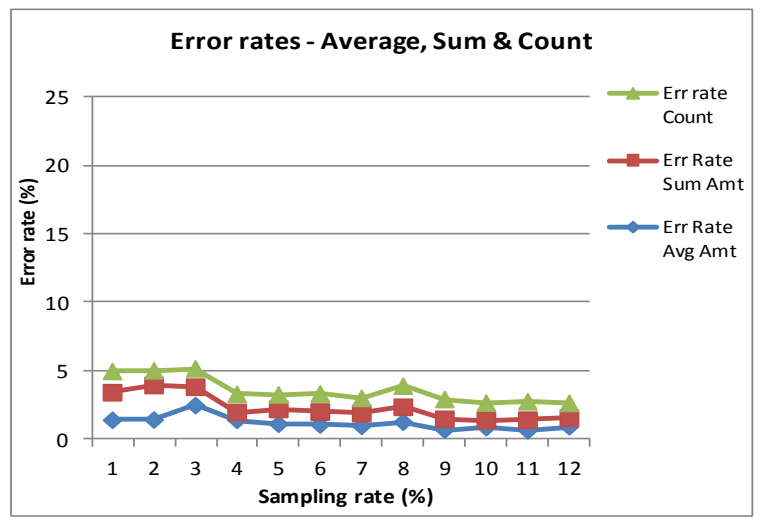

(a) Error rates for query with condition on one dimension (high relevancy ratio)

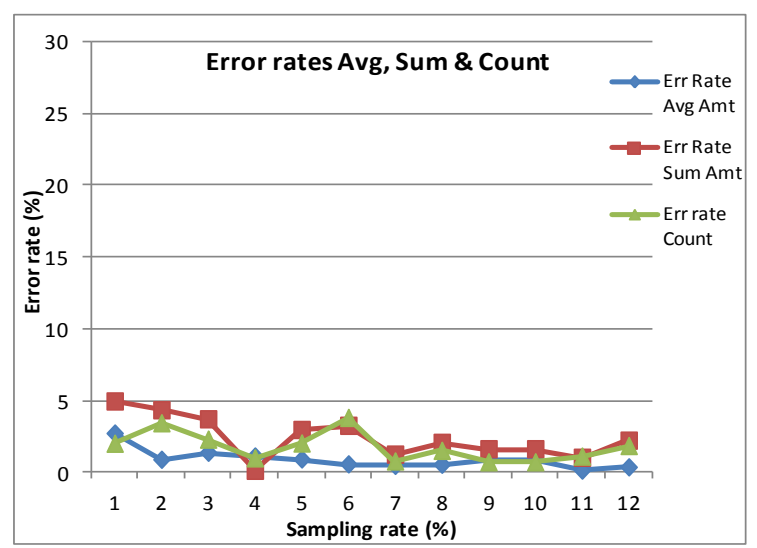

(c) Error rates for query with condition on two dimensions (medium relevancy ratio)

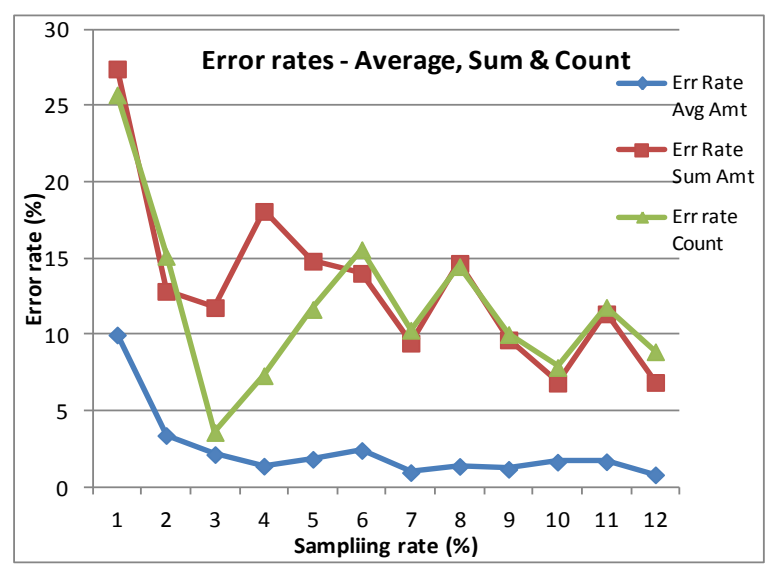

(e) Error rates for query with condition on three dimensions (low relevancy ratio)

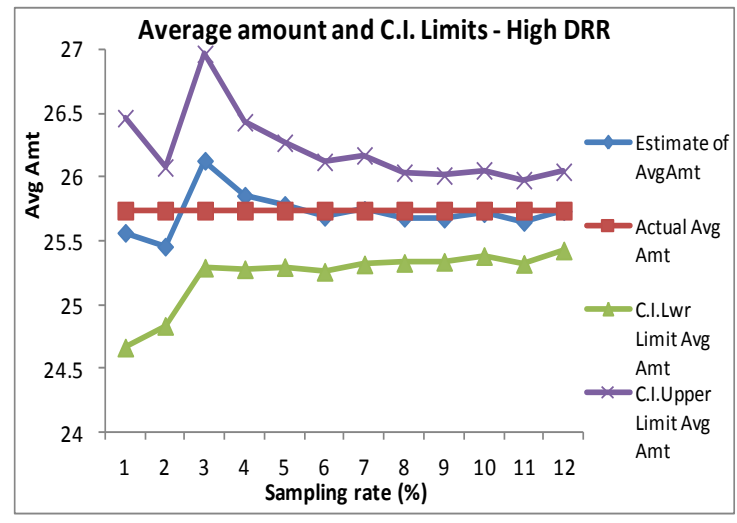

(b) Confidence interval (AVG amt) for query with condition on one dimension (high DRR)

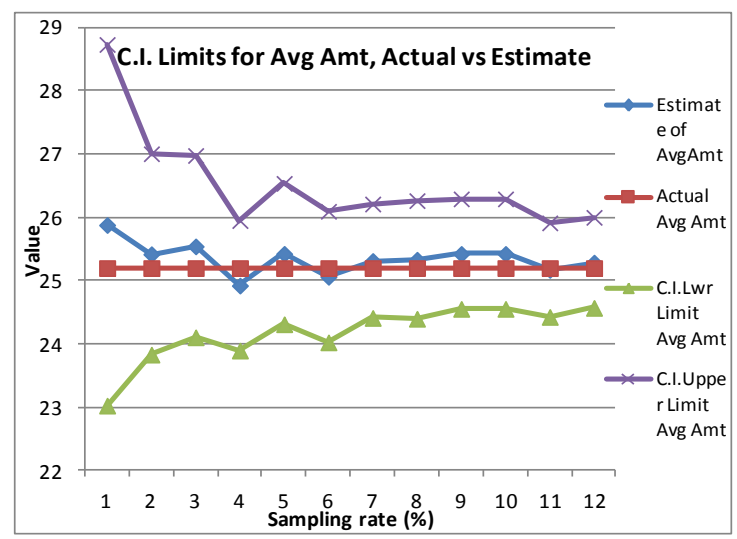

(d) Confidence interval (AVG amt) for query with condition on two dimensions (medium DRR)

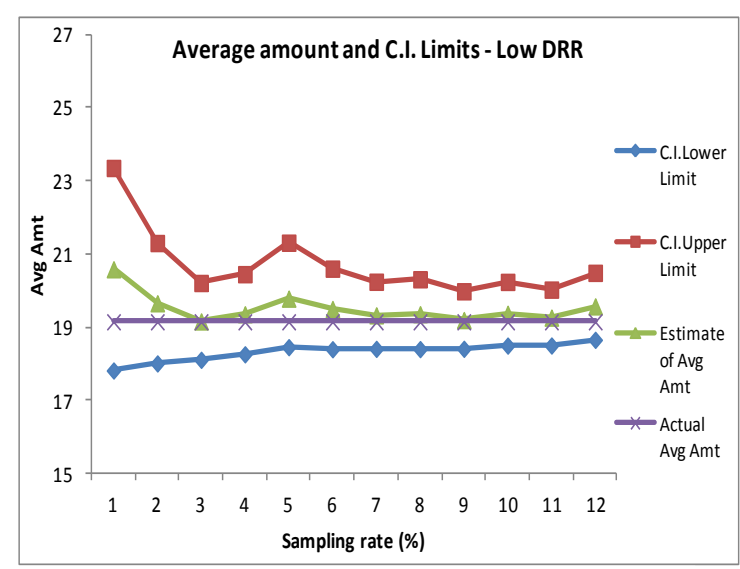

(f) Confidence interval for query with condition on three dimensions (low DRR)

Figure 6: Error rates of average scan amount and sum of scan amount and confidence interval of average scan amount at various sampling rates for high, medium and low relevancy ratios. 
Time Improvement - Figure 7 shows the average time for processing the queries at various sampling rates and also the average time for processing these queries on the full database. It is seen that there is a significant time improvement from using the sampling scheme.

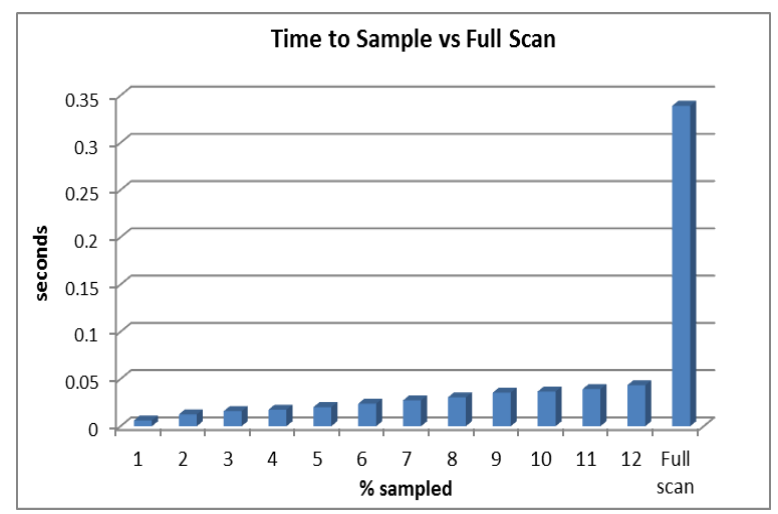

Figure 7: Query times at different sampling rates as compared to full database scan.

\section{CONCLUSIONS}

In this paper, we proposed the $k$-MDI tree index which can be used to draw samples quickly for answering multi-dimensional aggregate queries from a data warehouse. The $k$-MDI tree extends the ACE binary tree as a multi-way tree index. The maximum number of levels of the $k$-MDI index is limited to the number of key attributes and so makes the access to the leaf nodes much quicker compared to a binary tree index on external storage.

We also proposed the concepts of database relevancy ratio (DRR) and sample relevancy ratio (SRR) for queries. We investigated the effect of the DRR on the accuracy of query results estimated from samples drawn using the $k$-MDI index. From the experimental evaluation of the sampling scheme on a large real dataset, it is found that even at relatively low sampling rates of $1 \%$ to $12 \%$, query results can be estimated accurately with a minimum of $95 \%$ confidence for queries with medium to high DRR. At a very low DRR of 0.002 , the estimated values of sum and count fell outside the acceptable confidence level of $95 \%$, but the estimated mean was within the $95 \%$ confidence interval even at very low DRR. Depending on the sampling rate, the sampling based query processing was on average 9 to 30 times faster than processing the same queries against the whole dataset.
As future work, it is proposed to develop a generic tool that can be used with some parameter inputs to set up the $k$-MDI tree index for any data warehouse schema. We also plan to further evaluate the sampling based estimation scheme on data warehouses with larger dimensions.

\section{REFERENCES}

Acharya, S., Gibbons, P. B., Poosala, V., 2000. Congressional Samples for Approximate Answering of group-by Queries. 2000 SIGMOD Conference Proceedings., pp. 487-498.

Aluru, S., Prabhu, G. M., Gustafson, J., 1992. A Random Number Generator. Parallel Computing. NorthHolland, Elsevier Science Publishers, Amsterdam, The Netherlands, 18:9, pp.839-847.

Bain, L. J., Engelhardt, M., 1992. Introduction to Probability and Mathematical Statistics. $2^{\text {nd }}$ ed. PWSKent Publishing Co., Boston, USA.

Bentley, J. L., 1975. "Multidimensional binary search trees used for associative searching", Communications of the ACM, 18, 9, September 1975, pp. 509-517.

Bentley, J. L. and Shamos, M. I., 1976. Divide and Conquer in Multidimensional Space, Proceedings Eighth ACM.

Berenson, M. L., Levine, D. M., 1992. Basic Business Statistics - Concepts and Applications. Prentice Hall, Upper Saddle River, New Jersey, USA.

Chaudhuri, A., Mukherjee, R., 1985. Domain Estimation in Finite Populations. Australian Journal of Statistics. Vol. 27:2, pp. 135-137.

Hellerstein, H, Haas, P, Wang, J., 1997. Online Aggregation. SIGMOD 1997, pp. 171-182.

Hobbs, L., Hillson, S., Lawande, S., 2003. Oracle9iR2 Data Warehousing. Elsevier Scince, Burlington, MA, USA.

Jermaine, C., 2007. Random Shuffling of Large Database Tables. IEEE Transactions on Knowledge and Data Engineering. 18:1, pp.73-84.

Jermaine, C., 2003. Robust Estimation with Sampling and Approximate Pre-Aggregation. VLDB Conference Proceedings 2003, pp. 886-897.

Jermaine, C., Pol, A., Arumugam, S., 2004. Online Maintenance of Very Large Random Samples. SIGMOD Conference Proceedings 2004.

Jin, R., Glimcher, L, Jermaine, C, Agrawal, G., 2006. New Sampling-Based Estimators for OLAP Queries. Proceedings of the $22^{\text {nd }}$ International Conference on Data Engineering (ICDE'06), Atlanta, GA, USA.

Joshi, S., Jermaine, C., 2008. Matirialized Sample Views for Database Approximation, IEEE Transcaions on Knowledge and Data Engineering, 20:3 pp. 337-351.

Keller, G., 2009. Statistics for Management and Economics. Cengage Learning, Mason, OH, USA. 
Li, X., Han, J., Yin, Z., Lee, J-G., Sun, Y., 2008. Sampling Cube: A Framework for Statistical OLAP over Sampling Data. Proceedings of ACM SIGMOD International Conference on Management of Data (SIGMOD'08), Vancouver, BC, Canada, June.

Olken, F., Rotem, D., 1990, Random Sampling from Database File. In: A Survey. International Conference on Scientific and Statistical Database Management, 1990. pp. 92-111.

Raman, R., Raman, V., Rao, S. S., 2002. Succinct Indexable Dictionaries with Applications to Encoding $k$-ary Trees and Multisets, ACM-SIAM, pp. 233-242.

Sangngam, P., Suwatee, P., 2010. Modified Sampling Scheme in Inverse Sampling without Replacement. In: 2010 International Conference on Networking and Information Technology, pp. 580-584. IEEE Press, New York.

Seshadri, S. Naughton, J. F., 1995. Sampling Issues in a Parallel Database Systems. In: SIGMOD, 1995.

TPC Benchmarks, 2011. Transaction Processing Performance Council - TPC-H: Decision Support Benchmark. http://www.tpc.org [Accessed 20 November 2011].

TUN - Teradata University Network, 2011. http://www.teradata.com/TUN_databases. [Accessed: 13 April 2007].

Vitter, J. S., 1985. Random Sampling with a Reservoir. ACM Transactions on Mathematical Software. ACM Press, New York, NY, USA, 11:1, pp.37-57.

Wang, H., S. Parthasarathy, A. Ghoting, S. Tatikonda, G. Buehrer, T. Kurc, J. Saltz., 2005. Design of a Next Generation Samplng Service for Large Scale Data Analysis Applications. Proceedings of the ICS'05, Boston, MA, USA. pp. 91-100.

Zeeb, C. N., Burns, P. J., 2011. Random Number Generator Recommendation. Colorado State University.

http://www.colostate.edu/ pburns/monte/rngreport.pdf [Accessed 25 September 2011]. 